\title{
A Methodological Approach to Study Almond Oil Stability in Relation to $\alpha$-Tocopherol Supplementation
}

\author{
Lizette Auezova $^{1 *}$, Chawki Saliba ${ }^{1}$, Elie Hajj-Moussa ${ }^{1}$, Leina El Hosry ${ }^{2}$, Said Yammine ${ }^{3}$, \\ Ramez Chahine $^{4}$
}

${ }^{1}$ Department of Chemistry, Faculty of Sciences II, Lebanese University, Fanar, Jdaidet el Matn, Lebanon; ${ }^{2}$ Faculty of Pharmacy, Lebanese University, Hadath Campus, Beirut, Lebanon; ${ }^{3}$ Faculty of Agriculture and Veterinary Sciences, Lebanese University, Dekwaneh, Beirut, Lebanon; ${ }^{4}$ Faculty of Medical Sciences, Lebanese University, Hadath Campus, Beirut, Lebanon.

Email: *laouezova@ul.edu.lb

Received October 25 $5^{\text {th }}, 2012$; revised November $25^{\text {th }}$, 2012; accepted December $3^{\text {rd }}, 2012$

\begin{abstract}
The objectives of the present study were to identify a suitable parameter to assess the oil oxidation and to study the possibilities of delaying the oxidation of moderately oxidized unrefined almond oil by adding supplementary amounts of $\alpha$-tocopherol. Three oil samples, with initial peroxide values of 13.3, 19.5 (moderately oxidized oils), and 28.9 (oxidized oil), were supplemented with 100, 250 and $500 \mathrm{ppm}$ of $\alpha$-tocopherol and subjected to the oxidation at $50^{\circ} \mathrm{C}$ for 9 days. The level of oxidation was determined by measuring peroxide value (PV), conjugated dienes (CD) and thiobarbituric acid reactive substances (TBARS). Chemometric analysis indicated that, under the conditions of the present study, PV was the most suitable indicator of oil oxidation. The results have shown that the $\alpha$-tocopherol could have an antioxidant or pro-oxidant effects, depending on its concentration and initial level of oil oxidation. Results also show that $\alpha$ tocopherol addition at all the concentrations tested resulted in pro-oxidation of the moderately oxidized oils; hence additional supplementation with $\alpha$-tocopherol could not be recommended to delay oxidation of unrefined almond oil.
\end{abstract}

Keywords: Almond Oil; Oxidative Stability; $\alpha$-Tocopherol; PV; CD; TBARS; Chemometrics

\section{Introduction}

The oxidative stability of oil depends on its fatty acid composition, energy of heat or light, the concentration and type of oxygen, and the content of free fatty acids, mono- and diacylglycerols, transition metals, peroxides, thermally oxidized compounds, pigments, and antioxidants [1]. Lipid oxidation is a complex process; it involves the continuous formation of hydroperoxides as primary oxidation products that may break down to a variety of nonvolatile and volatile secondary products, providing various indices of lipid oxidation [2]. Among the most commonly used in food industry indices are peroxide value, conjugated dienes and thiobarbituric acid reactive substances. Peroxide value (PV) represents the total hydroperoxide content which is most frequently determined by iodometric titration. It is an indicator of the initial stages of oxidative change when the formation rate of hydroperoxides outweighs their rate of decomposition. During the formation of hydroperoxides from unsaturated fatty acids conjugated dienes (CD) are formed which absorb light at $234 \mathrm{~nm}$, so their content is also an indicator of the initial stages of oxidation. Degradation of

*Corresponding author. polyunsaturated fatty acids with 3 or more double bonds results in formation of malonaldehyde (MDA) that reacts with thiobarbituric acid (TBA) to form a pink complex with its absorption maximum at $530-535 \mathrm{~nm}$. However, TBA can react with many other substances such as alkenals and alkadienals and contribute to absorption; so the term "thiobarbituric acid reactive substances" (TBARS) is now used instead of MDA [3].

PV, CD and TBARS methods have been widely used to evaluate the effect of antioxidants on lipid oxidation. As each measurement method generates information only on part of the overall antioxidant activity, it has been strongly recommended to use various methods measuring both the primary and the secondary oxidation products [2]. On the other hand, a discrepancy between the results obtained on primary and secondary oxidation products formation in the presence of antioxidant was noted by various authors [4-7]. The discrepancy could be solved by processing the data obtained through chemometric analysis [7].

Alpha-tocopherol is the predominant fat-soluble antioxidant in human and animal tissues [8,9]. Tocopherols are also the most important antioxidants present in vegetable oils [10]. They act as radical scavengers competing 
with unsaturated oils for lipid peroxy radicals [1]. The antioxidant potential of tocopherols is related to there isomers composition and content. The optimal concentration of tocopherols isomers is dependent on their oxidative stability. Alpha-tocopherol is less stable than $\gamma$ - and $\delta$-tocopherols; therefore, it achieve maximum antioxidant activity at lower concentrations compared to other isomers [11,12]. By contrast, relatively high concentrations of tocopherols accelerate lipid oxidation; a phenomenon called "tocopherol mediated peroxidation" [13].

Sweet almond tree (Prunus dulcis) is widely cultivated in the Middle East for its delicious nuts rich in essential dietary nutrients as well as its oil. Almond oil is characterized by a very mild, sweet, nutty odor and prized by aromatherapists as one of the best carrier oils; it is also used as component in cosmetic products. However, almond oil is prone to rapid oxidation due to its high unsaturated fatty acid composition. Almond oil is a rich source of $\alpha$-tocopherol which was shown to contribute to the oil stability [14]. Namely, its content decreased progressively with parallel rise in peroxide value level, and became totally depleted after three years when stored at $4^{\circ} \mathrm{C}$. A similar, but faster, trend was observed in almond oil stored at ambient temperature. Complete consumption of $\alpha$-tocopherol at the end of the induction period was observed in different lipid substrates, clearly showing the apparent relationship between the disappearance of $\alpha$ tocopherol and the progress of oxidation [15-17]. A possibility of increasing the oxidative stability of moderately oxidized unrefined almond oil by addition of $\alpha$-tocopherol has not been studied yet.

The objectives of the present study were to identify a suitable parameter to assess the oil oxidation and to study the possibilities of delaying the oxidation of moderately oxidized unrefined almond oil by adding supplementary amounts of $\alpha$-tocopherol. Three oil samples with different initial degrees of oxidation were oxidized at $50^{\circ} \mathrm{C}$ for 9 days, in the presence of 100,250 , and $500 \mathrm{ppm}$ of $\alpha$-tocopherol. The evolution of oil oxidation was monitored at two days interval by measuring PV, CD and TBARS contents, and the results were treated by chemometric methods.

\section{Materials and Methods}

\subsection{Materials and Chemicals}

Unrefined sweet almond oil was obtained from BioCham Laboratories (Damascus, SAR). The major fatty acids were 16:0 (6.30\%), 18:0 (0.39\%), 18:1 (69.04\%), 18:2 (22.50\%), and 18:3 (0.30\%), and these contents were in accordance with those reported in the literature [14]. The almond oil content in $\alpha$-tocopherol was $280 \mathrm{mg} / \mathrm{kg}$ of oil. Peroxide value of the oil was $13.3 \mathrm{meq} \mathrm{O}_{2} / \mathrm{kg}$ oil. Two other oil samples were obtained by oxidation of the original oil at $50^{\circ} \mathrm{C}$ to achieve the peroxide values of 19.5 and 28.9 meq $\mathrm{O}_{2} / \mathrm{kg}$ oil, respectively.

Alpha-tocopherol and 2-thiobarbituric acid (TBA) were purchased from Sigma-Aldrich Co. (St. Louis, MO, USA). All reagents and solvents were of analytical grade.

\subsection{Oven-Oxidation Test}

Almond oil samples with or without added $\alpha$-tocopherol were transferred to glass sample vials and loosely capped before being placed in an oven at $50^{\circ} \mathrm{C}$ for 9 days. For each oil sample, five vials ( $20 \mathrm{~mL}$ each one) were loaded into the oven, and one was removed every 2 or 3 days for analysis. The oxidative state of oil was analyzed by measuring the peroxide value (PV), conjugated dienes (CD) and thiobarbituric acid reactive substances (TBARS).

\subsection{Chemical Analyses}

Fatty acid composition was determined by capillary gas chromatography with flame-ionization detection of the methyl esters prepared by transesterification using $\mathrm{BF}_{3} /$ methanol [18]. Alpha-tocopherol was measured by highperformance liquid chromatography (HPLC) according to the AOCS [19]. A Varian Vista 5500 HPLC system with fluorescence detector and a LiChrosorb Si-60 $(250 \times 4$ mm, $5 \mu \mathrm{m}$ particle size) column (Merck, Darmstadt, Germany) were used. Peroxide values (PV) were determined according to the AOCS [20]. Conjugated dienes (CD) content was determined by dissolving weighed oil samples $(0.05$ to $0.1 \mathrm{~g})$ in isooctane and reading the sample absorbance at $234 \mathrm{~nm}$. The $\mathrm{E}_{1 \mathrm{~cm}}^{1 \%}$ was calculated following an IUPAC method [21]. Thiobarbituric acid reactive substances (TBARS) content was determined according to Farag et al. [22]. An oil sample (0.75 g) was mixed with TBA $0.02 \mathrm{M}$ solution $(3 \mathrm{~mL})$ and benzene $(10 \mathrm{~mL})$ and vortexed for $30 \mathrm{~s}$. After shaking, the aqueous phase of the mixture was separated and heated in boiling water for 30 min. The absorbance of the resultant colored solution was recorded at $532 \mathrm{~nm}$. All determinations were performed in duplicate.

\subsection{Statistical Analysis}

Statistical analyses included a descriptive analysis for the three parameters assessing the degree of oil oxidation. Pearson coefficients of correlation evaluating the linear relationships among the three parameters were determined. To identify the most suitable indicator of oil oxidation, a PCA (principal component analysis) was carried out, as well as a correlation analysis assessing the relationships between the oxidation variables and the first principal component. The PV, selected as the most suitable indicator of oxidation was used in regression analysis to assess the effectiveness of added $\alpha$-tocopherol. All the statistical analyses were carried out using the statistical analysis software package SPSS, version 17. 


\section{Results and Discussion}

The almond oil samples with peroxide values 19.5 and 28.9 meq $\mathrm{O}_{2} / \mathrm{kg}$ oil (oils 2 and 3, respectively) were prepared by oxidation of the original oil (PV 13.3 meq $\mathrm{O}_{2} /$ $\mathrm{kg}$ oil; oil 1) at $50^{\circ} \mathrm{C}$. Based on PV of 20 meq $\mathrm{O}_{2} / \mathrm{kg}$ oil as a typical endpoint of oil oxidation [23,24], the oil samples 1 and 2 can be considered as moderately oxidized oils, while oil 3 with PV of 28.9 meq $\mathrm{O}_{2} / \mathrm{kg}$ oil was out of the range; so the latter was studied in a theoretical purpose. All the three oils, with or without added $\alpha$-tocopherol, were subjected to an accelerated oxidation and analyzed for PV, CD and TBARS contents.

The PV evolution for the three oils without added $\alpha$ tocopherol (control oils) is presented in Figure 1(a). All the oil samples showed a similar trend of changes in the PV with oxidation time. The peroxide values constantly increased during the first 5 days of oxidation, and then this trend was followed at a lower rate till the end of the oxidation assay; after 9 days of storage the peroxide values were seen to be between 42.7 and 54.0 meq $\mathrm{O}_{2} / \mathrm{kg}$ oil. $\mathrm{CD}$ values also displayed an increasing trend over storage time; a regular pattern of rise was observed for all the control oils, with values ranging from 6.05 to 7.60 at the end of oxidation (Figure 1(b)). Similarly, comparative analysis of TBARS values (Figure 1(c)) clearly demonstrated a direct relationship between the extent of oxida-

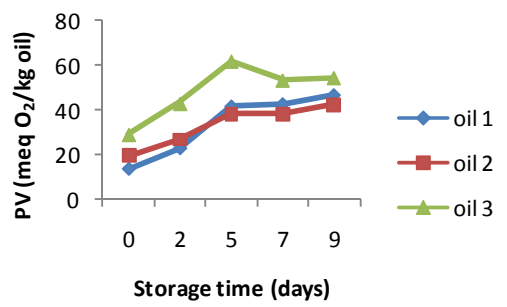

(a)

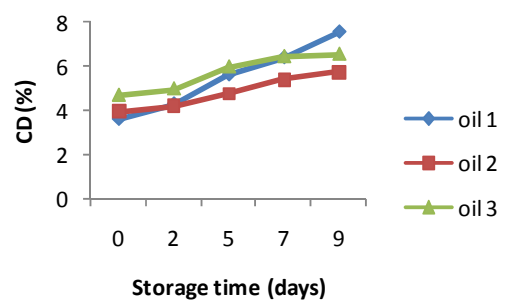

(b)

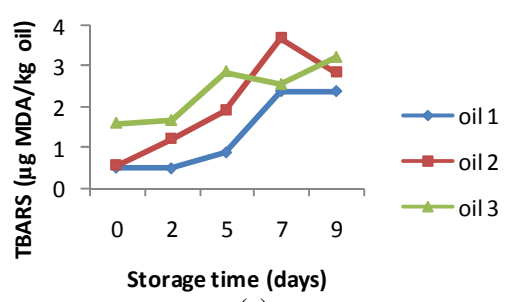

(c)

Figure 1. Evolution of PV (a), CD (b) and TBARS (c) of three control almond oils stored at $50^{\circ} \mathrm{C}$ for 9 days (Values are means of duplicate determinations). tion of the oils and their TBARS concentrations; their values on day 9 were between 2.40 to $3.23 \mu \mathrm{g}$ MDA $/ \mathrm{kg}$ oil.

However, the results obtained by the three methods on the effectiveness of $\alpha$-tocopherol addition (100, 250, and $500 \mathrm{ppm}$ ) were different. Namely, the formation of TBARS was inhibited by $\alpha$-tocopherol at all the doses tested in all the three oils, a typical example is shown in Figure 2 where the TBARS levels measured in presence of 500 $\operatorname{ppm} \alpha$-tocopherol are compared to the values obtained for the control oils during 9 days of oxidation. For example, on day 9 the oils 1 , 2, and 3 supplemented with $500 \mathrm{ppm}$ of $\alpha$-tocopherol contained 1.60, 1.76, and 1.45 $\mu \mathrm{g} \mathrm{MDA} /$ $\mathrm{kg}$ while the control oils were more oxidized (2.40, 2.87, and $3.23 \mu \mathrm{g}$ MDA/ kg, respectively). Moreover this inhibition was concentration-dependant; the effect of 100, 250 and $500 \mathrm{ppm} \alpha$-tocopherol on the oxidative stability of oil 1 is shown in Figure 3, as example.

In contrast, the effect of $\alpha$-tocopherol, based on hydroperoxide formation, was not clear. In order to understand the obtained results, a chemometric analysis was applied to treat the data collected over a total of 360 values (60 samples of almond oil with different degrees of oxidation). Table 1 shows few descriptive parameters of three analyzed variables, PV, CD and TBARS. The peroxide values ranged between 13.33 for the control oil and 63.20 for the most oxidized one. These values were 3.63 and 7.90 using the $\mathrm{CD}$, and for TBARS the values ranged between 0.31 and 3.55. The results of the correlation analyses among the three variables used to measure the oil oxidation are presented in Table 2. High positive correla-

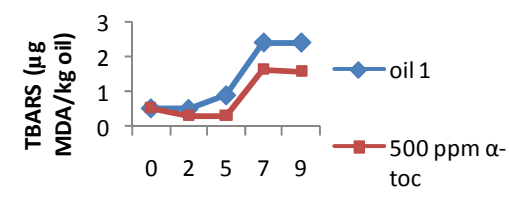

(a)

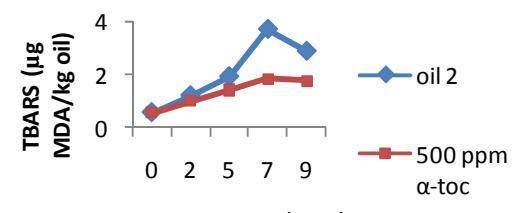

(b)

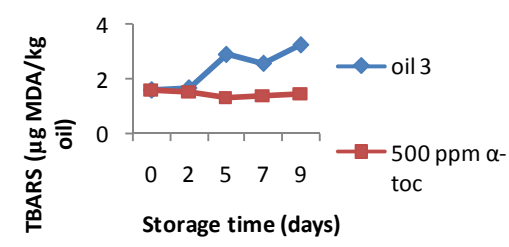

(c)

Figure 2. Effect of $500 \mathrm{ppm} \boldsymbol{\alpha}$-tocopherol, based on TBARS formation, to the oils with initial PV 13.3 (oil 1), 19.5 (oil 2) and 28.9 (oil 3), oxidized at $50^{\circ} \mathrm{C}$ for 9 days. 


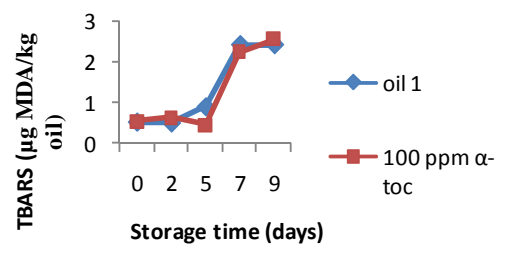

(a)

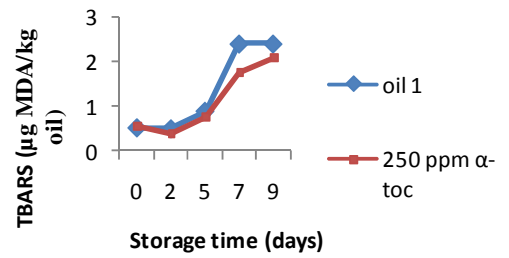

(b)

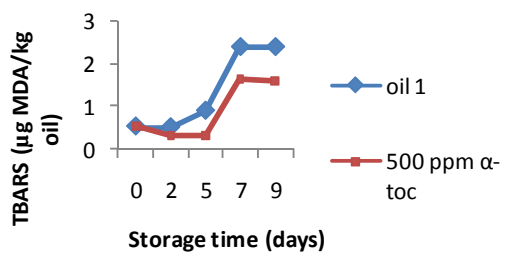

(c)

Figure 3. Effect of 100, 250, $500 \mathrm{ppm} \alpha$-tocopherol, based on TBARS formation, on the oil with initial PV 13.3 (oil1) oxidized at $50^{\circ} \mathrm{C}$ for 9 days.

Table 1. Descriptive statistics for the 3 variables, PV, CD and TBARS, evaluated on 60 almond oil samples with different degrees of oxidation.

\begin{tabular}{ccccc}
\hline Variable $^{*}$ & Mean & SE $^{* *}$ & Min & Max \\
\hline PV & 41.61 & 1.25 & 13.33 & 63.20 \\
CD & 5.55 & 0.11 & 3.63 & 7.90 \\
TBARS & 1.58 & 0.07 & 0.31 & 3.55 \\
\hline
\end{tabular}

*Measured in duplicate, ${ }^{* *}$ SE: Standard Error.

tions can be noted among all the variables, where the correlation between PV and CD is the highest (Figure 4). Significant correlations between peroxide value and conjugated dienes have been also found in other lipid substrates $[15,16,25]$. However, CD method has less specificity and sensitivity than PV measurement [25,26].

To reduce the oxidation dataset and to establish a new variable that could summarize the three observed oxidation variables (PV, CD and TBARS), a principal component analysis (PCA) was carried out. Table 3 shows the PCA results; the first component accounted for more than $83 \%$ of the total variances. The coefficients of correlation between the first component and each one of the three variables are all highly significant (Table 4) but the highest value was obtained for PV which therefore could be considered as the best indicator of oxidation. Similarly, Hung and Slinger [27] showed that among four analytical methods, PV, AV (Anisidine Value), TBARS and FFA (percent free fatty acids), applied for measuring the oxi-
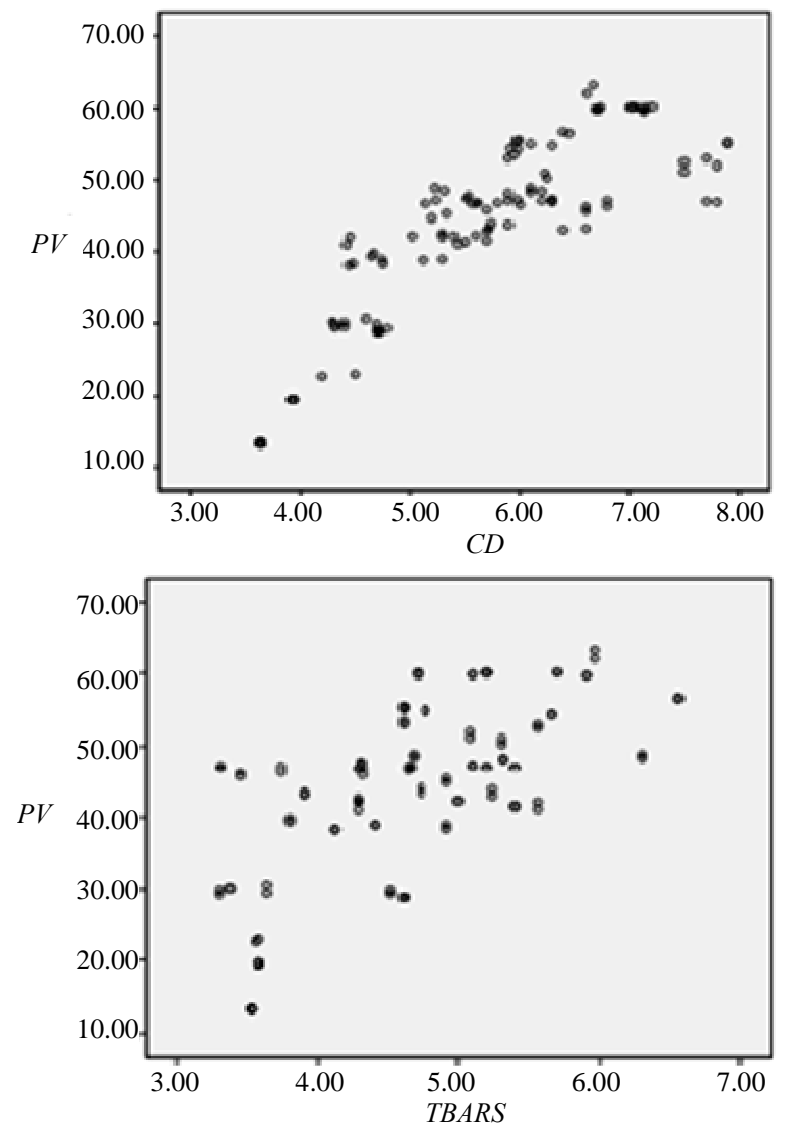

Figure 4. Scatter diagrams with PV as dependent variable and CD and TBARS as independent variables evaluated on 60 samples of almond oil.

Table 2. Coefficients of correlation among PV, CD and TBARS analyzing the data obtained from 60 samples of almond oil with different degrees of oxidation.

\begin{tabular}{ccc}
\hline & PV & CD \\
\hline CD & $0.88^{*}$ & \\
TBARS & 0.70 & 0.63 \\
\hline
\end{tabular}

*All coefficients of correlation are significant at a level less than 0.000 .

Table 3. Results of the principal components analysis; percentages of variances explained in the 3 variables evaluated on 60 samples of almond oil with different degrees of oxidation.

\begin{tabular}{cccc}
\hline Component & Eigen value & \% of variance & Cumulative \% \\
\hline 1 & 2.5 & 83 & 83 \\
2 & 0.4 & 14 & 97 \\
3 & 0.1 & 3 & 100 \\
\hline
\end{tabular}

Table 4. Correlation coefficients of the first principal component with the 3 variables PV, CD, TBARS, evaluated on 60 oil samples with different degrees of oxidation.

\begin{tabular}{cccc}
\hline & PV & CD & TBARS \\
\hline Principal component & 0.96 & 0.93 & 0.85 \\
\hline
\end{tabular}


dative stability of soybean oil, salmon oil, canola oil and canola soap stocks, only PV was highly correlated with aeration time in all the oils. One disadvantage of PV method largely evoked in the literature is its limited use to the earlier stages of lipid oxidation because the final stages are also characterized by low peroxide values. Based on these results, among the three analyzed methods, PV, CD, and TBARS, used to evaluate the oxidation level of almond oil during storage at $50^{\circ} \mathrm{C}, \mathrm{PV}$ was shown to be the most suitable.

Therefore, the efficiency of the $\alpha$-tocopherol as antioxidant was assessed using PV as indicator of oil oxidation. Table 5 shows the slope values of the oxidation process based on PV measurement, in function of the initial level of oil oxidation and the added amount of $\alpha$-tocopherol. A clear trend could be noted: the less the oil is oxidized, the faster is its oxidation in presence of $\alpha$-tocopherol. Thus, the effect of $\alpha$-tocopherol was prooxidant relative to the moderately oxidized oils 1 and 2, and this prooxidant effect was the most pronounced in the least oxidized oil 1 at maximal dose of $500 \mathrm{ppm}$ of $\alpha$-tocopherol.

By contrast, $\alpha$-tocopherol was rather without effect on the most oxidized oil 3 (PV 28.9 meq $\mathrm{O}_{2} / \mathrm{kg}$ oil). In other words, the effect of $\alpha$-tocopherol was shown to be dependent on the initial degree of oil oxidation (hydroperoxide concentration) and on the concentration of $\alpha$-tocopherol.

Previous studies on the effect of $\alpha$-tocopherol addition to oils indicated a strict dependence of the oxidation pattern on its concentration [15-17,28]. In addition, the rates of consumption of $\alpha$-tocopherol were strongly correlated with the amount of tocopherol added [8]. Evans et al. [29] determined the optimal concentrations for different tocopherols to inhibit soybean oil oxidation. The optimal concentration was determined for each type of tocopherol, and above their optimal concentrations, the tocopherols exhibited pro-oxidation behavior.

The apparent relationship between tocopherol concentration and its antioxidant activity may be influenced by the end-points selected to measure oxidation. This factor has contributed to the contradictory opinions in published literature regarding the concentrations necessary to stabi-

Table 5. Slopes of $P V=f(t)$ depending on the dose of $\alpha$-tocopherol added, for 60 almond oil samples with different degrees of oxidation

\begin{tabular}{ccccccc}
\hline \multirow{2}{*}{$\begin{array}{c}\text { Doses } \\
(\mathrm{ppm})\end{array}$} & \multicolumn{2}{c}{ Oil 1 } & \multicolumn{2}{c}{ Oil 2 } & \multicolumn{2}{c}{ Oil 3 } \\
\cline { 2 - 7 } & Slope & SE & Slope & SE & Slope & SE \\
\hline 0 & 8.76 & 1.07 & 7.01 & 0.45 & 7.53 & 1.12 \\
100 & 9.47 & 1.00 & 6.16 & 1.16 & 7.48 & 1.23 \\
250 & 9.30 & 1.07 & 5.80 & 1.16 & 7.54 & 1.16 \\
500 & 10.10 & 1.20 & 5.88 & 1.44 & 7.55 & 1.10 \\
\hline
\end{tabular}

lize oils. Thus, based on 20 meq $\mathrm{O}_{2} / \mathrm{kg}$ oil as end-point of oxidation, the results of Zuta et al. [30] on the oxidation of unrefined mackerel oil at $30^{\circ} \mathrm{C}$ in presence of $\alpha$-tocopherol, should be interpreted in another way. Namely, no antioxidant effect of 50 and $100 \mathrm{ppm} \alpha$-tocopherol added to the oil could be observed up to day 18 (PV 20); in other words, the addition of $\alpha$-tocopherol for oil stabilization in these experimental conditions should not be recommended.

\section{Conclusion}

The present study showed that among the three methods tested, PV, CD and TBARS, PV was the most suitable indicator of lipid oxidation; the use of this method alone is expected to be sufficient for monitoring oxidative stability of bulk oils. In addition, it is strongly recommended to apply the method at relatively low temperatures (up to $40^{\circ} \mathrm{C}-50^{\circ} \mathrm{C}$ ). The effectiveness of $\alpha$-tocopherol addition should be analyzed on the basis of at least three parameters, namely the tocopherol content of oil studied, the initial level of oil oxidation, and the time (end-point) of detection. From a practical point of view, the shelf-life of moderately oxidized unrefined almond oils $(\mathrm{PV}<20$ meq $\mathrm{O}_{2} / \mathrm{kg}$ oil) could not be extended by supplementary amounts of $\alpha$-tocopherol.

\section{Acknowledgements}

The authors are grateful to Dr Rania Jounblat for her valuable comments during the preparation of this manuscript. This study was financially supported by the Doctoral School of Science and Technology of Lebanese University.

\section{REFERENCES}

[1] E. Choe and D. B. Min, "Mechanisms and Factors for Edible Oil Oxidation," Comprehensive Reviews in Food Science and Food Safety, Vol. 5, No. 4, 2006, pp. 169186. doi:10.1111/j.1541-4337.2006.00009.x

[2] E. N. Frankel, "Methods to Determine the Extent of Oxidation,” In: Lipid Oxidation, 2nd Edition, The Oily Press, England, 2005, pp. 99-127.

[3] A. De las Heras, A. Schoch, M. Gibis and A. Fischer, "Comparison of Methods for Determining Malondialdehyde in Dry Sausage by HPLC and the Classic TBA Test," European Food Research and Technology, Vol. 217, No. 2, 2003, pp. 180-184. doi:10.1007/s00217-003-0728-X

[4] S. W. Huang, E. N. Frankel and J. B. German, "Effects of Individual Tocopherols and Tocopherol Mixtures on the Oxidative Stability of Corn Oil Triglycerides," Journal of Agricultural and Food Chemistry, Vol. 43, No. 9, 1995, pp. 2345-2350. doi:10.1021/jf00057a006

[5] A. M. Lampi, L. Kataja, A. Kamal-Eldin and V. Piironen, "Antioxidant Activities of $\alpha$ - and $\gamma$-Tocopherols in the 
Oxidation of Rapeseed Oil Triglycerides,” Journal of the American Oil Chemists' Society, Vol. 76, No. 6, 1999, pp. 749-755.

[6] U. Samotyja and M. Matecka, “Antioxidant Activity of Blackcurrant Seeds Extract and Rosemary Extracts in Soybean Oil,” European Journal of Lipid Science and Technology, Vol. 112, No. 12, 2010, pp. 1331-1336. doi:10.1002/ejlt.201000042

[7] M. Laguerre, J. Lecomte and P. Villeneuve, "Evaluation of the Ability of Antioxidants to Counteract Lipid Oxidation: Existing Methods, New Trends and Challenges,” Progress in Lipid Research, Vol. 46, No. 5, 2007, pp. 244-282. doi:10.1016/j.plipres.2007.05.002

[8] J. M. Tucker and D. M. Townsend, "Alpha-Tocopherol: Roles in Prevention and Therapy of Human Disease," Biomedicine \& Pharmacotherapy, Vol. 59, No. 9, 2005, pp. 380-387. doi:10.1016/j.biopha.2005.06.005

[9] J.-M. Zingg, "Vitamin E: An Overview of Major Research Directions," Review. Molecular Aspects of Medicine, Vol. 28, No. 5-6, 2007, pp. 400-422. doi:10.1016/j.mam.2007.05.004

[10] S. Arranz, R. Cert, J. Pérez-Jiménez, A. Cert, F. SauraCalixto, et al., "Comparison between Free Radical Scavenging Capacity and Oxidative Stability of Nut Oils,” Food Chemistry, Vol. 110, No. 4, 2008, pp. 985-990. doi:10.1016/j.foodchem.2008.03.021

[11] M. Y. Jung and D. B. Min, "Effects of $\alpha$-, $\gamma$-, and $\delta$-Tocopherols on Oxidative Stability of Soybean Oil," Journal of Food Science, Vol. 55, No. 5, 1990, pp. 1464-1465. doi:10.1111/j.1365-2621.1990.tb03960.x

[12] N. V. Yanishlieva, A. Kamal-Eldin, E. M. Marinova and A. G. Toneva, "Kinetics of Antioxidant Action of $\alpha$ - and $\gamma$-Tocopherols in Sunflower and Soybean Triacylglycerols," European Journal of Lipid Science and Technology, Vol. 104, No. 5, 2002, pp. 262-270. doi:10.1002/1438-9312(200205)104:5<262::AID-EJLT26 2>3.0.CO;2-B

[13] Y. Yamamoto, "Role of Active Oxygen Species and Antioxidants in Photoaging," Journal of Dermatological Science, Vol. 27, No. 1, 2001, pp. 1-4.

[14] F. Salvo, M. Alfa and G. Dugo, “Almond Oil Composition. Note III: Variation of Several Chemical and Physico-chemical Parameters during the Storage," Italian Journal of Fat Matters, Vol. 63, 1986, pp. 37-40.

[15] E. Kulås and R. G. Ackman, "Different Tocopherols and the Relationship between Two Methods for Determination of Primary Oxidation Products in Fish Oil," Journal of Agricultural and Food Chemistry, Vol. 49, No. 4, 2001, pp. 1724-1729. doi:10.1021/jf0011541

[16] M. Deiana, A. Rosa, C. Falqui Cao, P. M. Pirisi, G. Bandino and A. Dessi, "Novel Approach to Study Oxidative Stability of Extra Virgin Olive Oils: Importance of $\alpha$ Tocopherol Concentration,” Journal of Agricultural and Food Chemistry, Vol. 50, No. 15, 2002, pp. 4342-4346. doi:10.1021/jf020033t

[17] D. Krichene, A. Allalout, V. Mancebo-Campos, M. D. Salvador, M. Zarrouk and G. Fregapane, "Stability of Virgin Olive Oil and Behavior of Its Natural Antioxidants under Medium Temperature Accelerated Storage Conditions," Food Chemistry, Vol. 121, No. 1, 2010, pp. 171177. doi:10.1016/j.foodchem.2009.12.026

[18] S. Sigurgisladottir, C. C. Parrish, S. P. Lall and R. G. Ackman, "Effect of Feeding Natural Tocopherols and Astaxanthin on Atlantic Salmon (Salmo salar) Fillet Quality,” Food Research International, Vol. 27, No. 1, 1994, pp. 23-32. doi:10.1016/0963-9969(94)90174-0

[19] AOCS, "Official Methods and Recommended Practices of the American Oil Chemists' Society," 4th Edition, AOCS Press, Champaign, 1993.

[20] AOCS, "Official Methods and Recommended Practices of the American Oil Chemists' Society," 5th Edition, AOCS Press, Champaign, 1999.

[21] IUPAC, "Standard Methods for the Analysis of Oils, Fats and Derivatives,” 7th Edition, Blackwell Scientific, London, 1987.

[22] R. S. Farag, A. Z. Badel and G. S. A. El Baroty, "Influence of Thym and Clove Essential Oils on Cottonseed Oil Oxidation," Journal of the American Oil Chemists' Society, Vol. 66, No. 6, 1989, pp. 800-804.

[23] E. A. Decker, K. Warner, M. P. Richards and F. Shahidi, "Measuring Antioxidant Effectiveness in Food," Journal of Agricultural and Food Chemistry, Vol. 53, No. 10, 2005, pp. 4303-4310. doi:10.1021/jf058012x

[24] C. Zajdenwerg, G. F. Branco, J. Alamed, E. A. Decker and I. A. Castro, "Correlation Between Sensory and Chemical Markers in the Evaluation of Brazil Nut Oxidative Shelf-Life," European Food Research and Technology, Vol. 233, No. 1, 2011, pp. 109-116. doi:10.1007/s00217-011-1493-X

[25] S. Marmesat, A. Morales, J. Velasco, M. V. Ruiz-Méndez and M. C. Dobarganes, "Relationship between Changes in Peroxide Value and Conjugated Dienes during Oxidation of Sunflower Oils with Different Degree of Unsaturation,” Oils and Fats, Vol. 60, No. 2, 2009, pp. 155-160. doi:10.3989/gya.096908

[26] M. Chaijan, S. Benjakul, W. Visessanguan and C. Faustman, "Changes of Lipids in Sardine (Sardinella gibbosa) Muscle during Iced Storage,” Food Chemistry, Vol. 99, No. 1, 2006, pp. 83-91. doi:10.1016/j.foodchem.2005.07.022

[27] S. S. O. Hung and S. J. Slinger, "Studies of Chemical Methods for Assessing Oxidative Quality and Storage Stability of Feeding Oils," Journal of the American Oil Chemists' Society, Vol. 58, 1981, pp. 785-788.

[28] R. E. King, D. B. Min and S. C. Min, "Study of $\alpha$-, $\gamma$-, and $\delta$-Tocopherols in the Oxidative Stability of Lard," Food Science and Biotechnology, Vol. 20, No. 3, 2011, pp. 817-822. doi:10.1007/s10068-011-0113-7

[29] J. C. Evans, D. R. Kodali and P. B. Addis, "Optimal Tocopherol Concentrations to Inhibit Soybean Oil Oxidation," Journal of the American Oil Chemists' Society, Vol. 79, No. 1, 2002, pp. 47-51.

[30] P. C. Zuta, B. K. Simpson, X. Zhao and L. Leclerc, “The Effect of $\alpha$-Tocopherol on the Oxidation of Mackerel Oil," Food Chemistry, Vol. 100, No. 2, 2007, pp. 800-807. doi:10.1016/j.foodchem.2005.11.003 